REVIEW ARTICLE

\title{
Basic Fetal Heart Examination: The Protocol at 18-22 Weeks and Literature Review
}

\author{
Rafał Iciek ${ }^{1}$, Sara Królik ${ }^{2}$, Kinga Toboła-Wróbel ${ }^{3}$, Kinga Bednarek ${ }^{4}$, Marek Pietryga ${ }^{5}$, Jacek Brązert ${ }^{6}$
}

\begin{abstract}
Congenital heart defects (CHDs) remain the most common birth defect in live births and are the main factors of infant morbidity and mortality in the developed countries. Rapid development of technology over the past decade has led to a significant change in survival rates of infants with CHDs. Early diagnosis and appropriate CHD treatment are essential for the improvement of perinatal outcome. Each year in Poland, we observe dynamic progress in the use of ultrasound prenatal techniques, which offers pregnant women a high-level examination. Basic screening examinations are offered for majority of women, as well as, for selected cases who demand more advanced and extensive ultrasound including fetal echocardiography. The aim of this paper is to summarize the recent knowledge in fetal screening ultrasound of the heart as a standard in the daily practice of every obstetrician-gynecologist.
\end{abstract}

Keywords: Cardiac axis, Cardiac fetal scan, Congenital heart defects, Echocardiography, Prenatal ultrasonography, Ultrasound.

Donald School Journal of Ultrasound in Obstetrics and Gynecology (2020): 10.5005/jp-journals-10009-1657

\section{INTRODUCTION}

Congenital heart defects are the most common fetal structural anomalies, either isolated or in association with many different fetal anatomical abnormalities. Congenital heart defects are strongly associated with genetic syndromes and chromosomal anomalies and can significantly affect the clinical outcome in the newborns. The major aim of the fetal heart screening ultrasound is to exclude potential anomalies and to detect any present anatomical abnormalities in the fetus. However, the factors such as obesity in pregnancy, large and small amniotic fluid volume, extensive fetal movements, arrhythmias, and unfavorable fetal position are factors that impede heart evaluation.

The CHDs affect about 8-10 cases per 1000 live births. $190 \%$ of fetuses with congenital heart disease are in low-risk population, whereas only $10 \%$ present some risk factors for CHD such as type I diabetes or family history of CHDs. ${ }^{2}$ Fetal cardiac evaluation constitutes an important element of the ultrasound examination in the course of pregnancy. Ultrasound scan, especially in reference centers, enables precise detection of CHD and start of appropriate prenatal treatment as far as this clinical entity is concerned. Vital factors influencing the effectiveness of the ultrasound screening program include constant submission of qualifications and training of health professionals, easy access to fetal heart specialists, and more favorable indications for echocardiography. ${ }^{3}$ This approach helps to identify fetuses at high risk of genetic syndromes and provide useful information for obstetrical management. Every case of suspected fetal heart anomalies will require more comprehensive examination including fetal echocardiography in the reference center (Figs 1 and 2). ${ }^{4}$

Fetal ultrasound heart screening is performed between 18 and 22 weeks of pregnancy. ${ }^{3,5}$ Some fetal heart abnormalities can be detected between the late first and early second trimester, especially when fetal nuchal translucency (NT) is observed in the first trimester. ${ }^{3}$ If the scan is performed at 20-22 weeks, there is a greater probability that of complete fetal scan with no requirement of additional scans later pregnancy. ${ }^{5}$ Any abnormality in fetal
${ }^{1-6}$ Department of Obstetrics and Maternal Diseases, Karol Marcinkowski University of Medical Sciences, Poznań, Poland

Corresponding Author: Rafał Iciek, Department of Obstetrics and Maternal Diseases, Karol Marcinkowski University of Medical Sciences, Poznań, Poland, Phone: +48618419334, +603775134, e-mail: rafaliciek1979@gmail.com, kpichk@gpsk.am.poznan.pl

How to cite this article: Iciek R, Królik S, Toboła-Wróbel K, et al. Basic Fetal Heart Examination: The Protocol at 18-22 Weeks and Literature Review. Donald School J Ultrasound Obstet Gynecol 2020;14(3): 222-225.

Source of support: Nil

Conflict of interest: None

heart screening is an indication for fetal echocardiography at the reference center. ${ }^{2} \mathrm{~A}$ pregnant woman is referred to undergo echocardiographic scan at prenatal cardiology reference centersthe full list is available at www.orpkp.pl.

Factors which increase the risk of fetal cardiac anomalies include a family history of CHD, abnormalities detected at basic cardiac examination at first trimester, increased NT at first trimester, tricuspid regurgitation at first trimester, chromosomal anomalies in the fetus, monochorionic twin pregnancies, as well as pregnancies from assisted reproductive technologies (ARTs) (Table 1). ${ }^{6}$

Detection of some anomalies such as aortic stenosis at an early stage enables intrauterine treatment which can improve the perinatal outcome.

Detection of fetal heart rate (FHR) (with $\mathrm{CRL}>4 \mathrm{~mm}$ ) can be reported during vaginal ultrasound before 10 weeks of pregnancy. In the absence of FHR with a $C R L<5 \mathrm{~mm}$, a reexamination should be performed to confirm a normal development of pregnancy. During an ultrasound at $11-13^{+6}$ weeks of pregnancy, fetal heart action should be assessed. In a normal pregnancy, the FHR decreases from about 170 beats per minute at 11 weeks to 150 beats per minute at the 14th week of pregnancy.

Another goal of $11-13^{+6}$ weeks of pregnancy ultrasound is to assess the risk of the most common chromosomal aberrations

(c) The Author(s). 2020 Open Access This article is distributed under the terms of the Creative Commons Attribution 4.0 International License (https://creativecommons. org/licenses/by-nc/4.0/), which permits unrestricted use, distribution, and non-commercial reproduction in any medium, provided you give appropriate credit to the original author(s) and the source, provide a link to the Creative Commons license, and indicate if changes were made. The Creative Commons Public Domain Dedication waiver (http://creativecommons.org/publicdomain/zero/1.0/) applies to the data made available in this article, unless otherwise stated. 

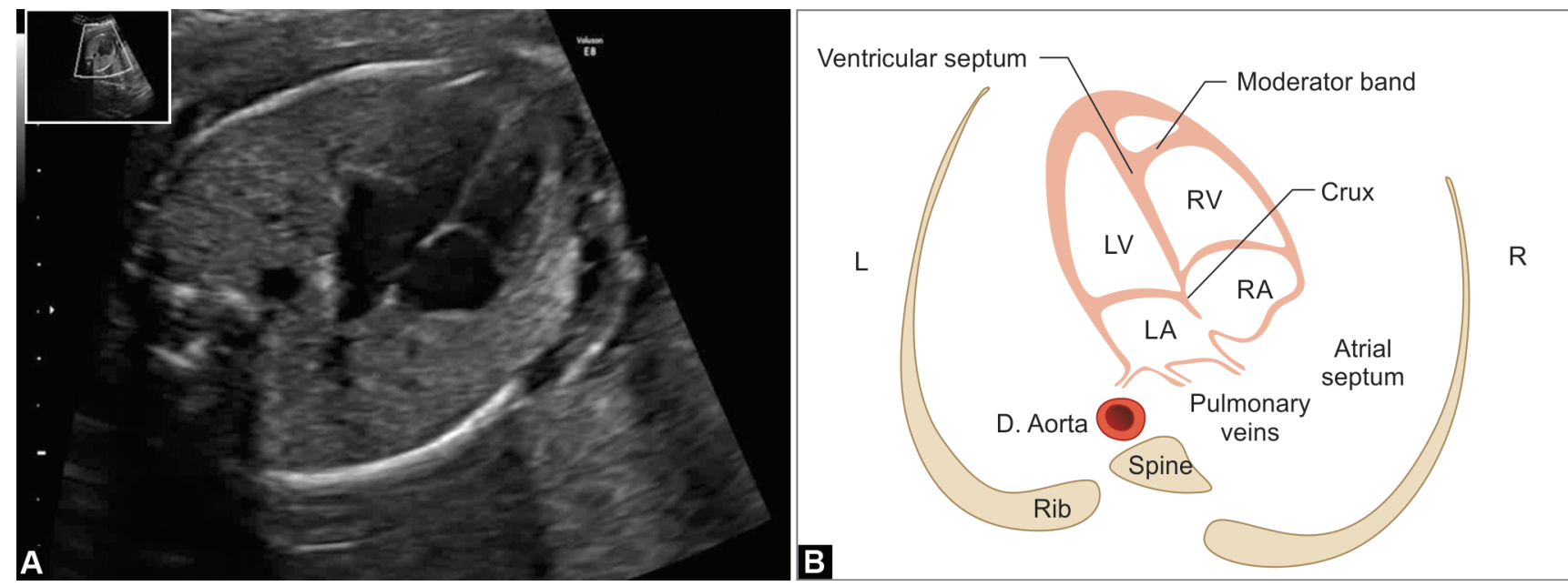

Figs $1 \mathrm{~A}$ and B: Four-chamber view of the fetal heart: (A) Ultrasound scan; (B) Figure ${ }^{5}$
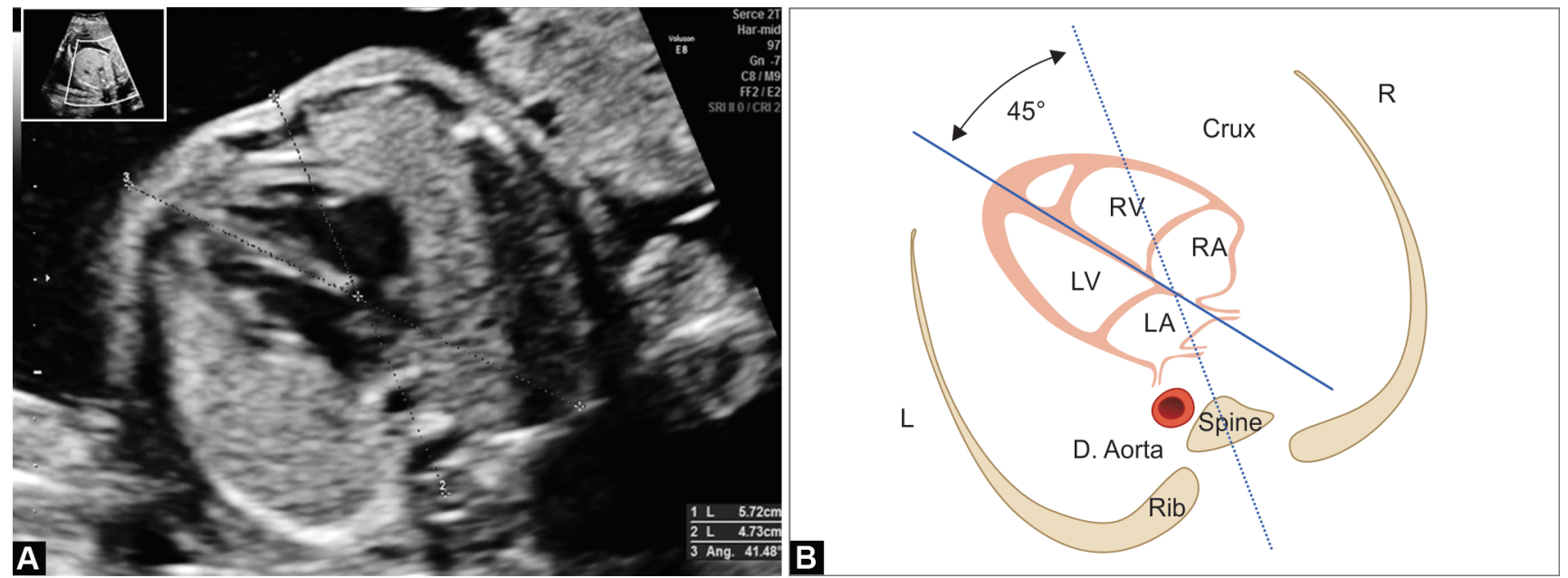

Figs 2A and B: Fetal cardiac axis and position. The cardiac axis can be measured in a four-chamber view of the fetal heart: (A) Ultrasound scan; (B) Figure

Table 1: Risk factors associated with the probability of $\mathrm{CHDs}^{6}$

\begin{tabular}{ll}
\hline Indication & Association with cardiac defects \\
\hline Noncardiac structural anomalies & $21 \%$ \\
Previous history of CHD & $8.7 \%$ \\
Abnormal ductus venosus & $7.5 \%$ \\
Increased NT & $7 \%$ \\
Monochorionic twins & $5.5 \%$ (9.3\% in cases with TTS) \\
Tricuspid regurgitation & $5.1 \%$ \\
Aberrant right subclavian artery & $5.1 \%$ \\
Consanguinity & $4.4 \%$ \\
ARTs & $4.3 \%$ \\
\hline
\end{tabular}

(trisomies 13, 18, 21). Risk calculation is based on maternal age and history, and assessment of ultrasound and biochemical markers. Ultrasound factors include FHR and NT. The purpose of an ultrasound examination at 18-22 and 28-32 weeks of pregnancy is a detailed assessment of fetal organs for congenital malformations (fetal anomaly scan). The protocol for basic fetal heart examination at $18-22$ weeks is presented in Table 2.

It is recommended to zoom the heart image so that it occupies $1 / 2$ or $1 / 3$ of the screen. ${ }^{7}$ Usual, basic fetal heart examination relies on a four-chamber view of the fetal heart. In some cases, a small hypoechogenic rim around the fetal heart can be mistaken for a pericardial effusion which can be misleading. ${ }^{3,5}$ In most cases, this isolated finding is a normal variation.

Normal four-chamber view consists of intact intraventricular septum and atrial septum primum. In most cases, the four-chamber view and outflow tract views can be identified from 12 weeks of gestation. ${ }^{6}$ There is no disproportion between right ventricle (RV) and left ventricle (LV). A moderator band helps to recognize the morphology of a right ventricle on the same side of the heart. ${ }^{3}$

Both atria usually look similar in terms of size. The foramen ovale valve should open to the left atrium. Four pulmonary veins end in the left atrium, usually two of four can be visualized. However, their identification should not be considered as a compulsory part of a basic fetal heart scan. The lower rim of the atrial septum tissue identified as the primum septum should be present. The moderator band helps to identify the morphology of a right ventricle. Both ventricles should also look similar in size without evidence of thickened walls. Mild ventricular imbalance may occur as a normal variant, as hypoplastic left heart syndrome (HLHS) and aortic stenosis (AS) are the most common causes of this imbalance. ${ }^{3}$

The ventricular septum should be carefully examined for cardiac wall defects from the apex to crux. Septal wall defects 
Table 2: Protocol for basic fetal heart examination at 18-22 weeks ${ }^{5}$

Step-by-step protocol
Identification of fetal sides based on its location in the uterus
Visualization of the gastric bulb on the left side
Visualization of the descending aorta to the left and in front of the
fetal spine
Visualization of the inferior vena cava forward from the aorta and
on the right of the fetal spine
Visualization of the heart in the chest
Heart size-the area of the heart is about $1 / 3$ of the area of the
chest
Determination of the cardiac axis-45 $\pm 20^{\circ}$
No effusion in the pericardial sac
Determination of FHR-110-150 beats/min, sinus rhythm
Visualization of four chambers of the heart (4CHV) with the heart
crux
Visualization of three vessels view in the upper mediastinum
(3VV)-pulmonary trunk, aorta, and superior vena cava
Visualization of three vessels and the trachea view in the upper
mediastinum ( $3 V T$ ) - the trachea to the right of the aortic arch and
arterial duct
Visualization of the outflow tracts from the ventricles
Visualization of the left ventricle outflow tract-LVOT
Visualization of the right ventricle outflow tract-RVOT
Visualization of the Ao and PA intersection after leaving the appro-
priate ventricles

can be difficult to detect when the transducer insonation angle is directly parallel to the ventricle wall. In such circumstances, a defect can be suspected due to the acoustic "drop-out" artifact. Small septal defects $(1-2 \mathrm{~mm})$ may be difficult to detect if the ultrasound imaging system does not provide a sufficient degree of lateral resolution, especially if the size and position of the fetus are unfavorable. Two separate atrioventricular valves (right-sided, tricuspid and left-sided, mitral) should be opened separately and freely. Incorrect atrioventricular valve positioning may be a key ultrasound finding for heart defects such as ventricular septal defect. ${ }^{3}$

Moreover, it is important to mention about diagnostic traps when examining a fetal heart in the four-chamber view. Some changes in a heart are difficult to detect until late pregnancy and the four-chamber view does not always allow clear visualization of the specific anomalies (transposition of the great arteries or aortic coarctation). ${ }^{3}$

\section{Ultrasound Findings Suggestive of Fetal Cardiac Anomalies}

\section{Heart Size}

The relationship between a heart and a thorax is the main parameter related to cardiac anomalies. An abnormal heart-to-chest area ratio $>0.28$ at $11-13^{+6}$ weeks of gestation may suggest cardiomegaly and a possible cardiac anomaly. ${ }^{6}$

Fetal heart size should be included in the echocardiographic report because of its clinical value. Cardiomegaly, if associated with CHD, usually affects long-term prognosis, often with an unfavorable result and perinatal outcome. For growth-restricted fetuses, cardiomegaly was one of the strongest prognostic factors of adverse neonatal outcomes. In addition, in severe twin transfusion syndrome in the second trimester of pregnancy, cardiomegaly is one of the most frequently observed features, more often in the recipient twin, usually lethal if untreated. The cardiothoracic ratios are also useful in the prenatal assessment of pulmonary hypoplasia and skeletal dysplasia. Therefore, detection of fetal heart cardiomegaly can have a significant impact on neonatal observation and parental counseling. ${ }^{8}$

\section{Cardiac Rhythm}

Rate and regular rhythm of the fetal heart should be evaluated. Normal heart rate varies between 120 and 160 beats per minute. Mild bradycardia is observed in fetuses at the end of the second trimester. When the heart rhythm decreases below 110 beats per minute, it is indicative of a fixed bradycardia, and a diagnosis toward fetal heart block should be introduced. Repeated decelerations in heart rate during the third trimester may be caused due to a possible fetal distress. If the heart rate exceeds 160 beats per minute, it is suggestive of a mild tachycardia, which usually occurs during fetal movements. However, when persistent tachycardia does not pass, it should be further evaluated for more serious tachydyshythmias or possible fetal distress. ${ }^{5}$

\section{Nuchal Translucency}

An increased NT is a serious ultrasound finding which may suggest the cardiac defect. An increased NT thickness above $3.5 \mathrm{~mm}$ at $11-13^{+6}$ weeks is an indication for a detailed cardiac evaluation. ${ }^{5}$ Iliescu et al. ${ }^{9}$ and Becker and Wegner ${ }^{10}$ reported that fetuses with increased NT had major cardiac anomalies. Former ${ }^{9}$ observed that $8.7 \%$ of fetuses with increased NT developed cardiac anomalies10 -fold higher than in a normal group. The latter ${ }^{10}$ reported that fetuses with NT (>2.5 mm) had $9.8 \%$ prevalence of heart defects, whereas group with a normal translucency had $0.3 \%$ prevalence of heart abnormalities.

\section{Cardiac Axis}

Abnormal deviation of the cardiac axis may occur in fetuses with heart defects, especially in the outflow tract. This finding may be associated with a chromosomal anomaly. Sinkovskaya et al. ${ }^{11}$ reported that $74.1 \%$ of fetuses with $\mathrm{CHD}$ had an abnormal cardiac axis during evaluation between 11 and $13^{+6}$ weeks of gestation. The same group observed a normal variation in the cardiac axis which fluctuated from $34.5^{\circ}$ at 11 weeks to $56.8^{\circ}$ at $13^{+6}$ weeks of gestation, and it can be associated with some anomalies such as heterotaxy, Ebstein's anomaly, coarctation of the aorta, and transposition of the great vessels. ${ }^{12}$

Some hearts are abnormally displaced from their usual position in the anterior left central chest. An abnormal heart position can be caused by the presence of congenital diaphragmatic hernia or a space-occupying lesion, such as a cystic adenomatoid malformation. Position abnormalities may also be secondary to fetal lung hypoplasia or agenesis.

\section{Abnormal Ductus Venosus}

Borrell et al. ${ }^{13}$ discovered that $39 \%$ of fetuses with CHD evaluated at $11-13^{+6}$ weeks of gestation had a reversed atrial waveform in the ductus venosus. Another study performed by Pereira et al. ${ }^{14}$ studied $85 \%$ euploid fetuses with CHD during the first trimester ultrasound scan and reported increased NT (>95th percentile) in $35.3 \%$, tricuspid regurgitation in $32.9 \%$, and reversed $A$ wave in the ductus venosus in $28.2 \%$ of them. 
The knowledge of the structure of the heart and the physiology of fetal circulation can change the natural course of many congenital anomalies in newborns and children. During pregnancy with fetal heart disease, the following symptoms may appear: circulatory failure, arrhythmias, and infection. These are factors adversely affecting the prognosis, which sometimes force obstetricians to terminate pregnancy prematurely, which is usually associated with increased risk of neonatal death.

\section{When is the Optimal Time to Perform an Early Fetal Cardiac Evaluation?}

The four-chamber view and outflow tracts can be visualized in the majority of fetuses after 12 weeks, whereas at the 11th week of gestation, these structures can be seen in only $20-37 \%$ of fetuses. ${ }^{15,16}$ Moreover, Smrcek et al. ${ }^{17}$ observed successfully cardiac structures starting at the 10th week of gestation and obtained the following planes: the four-chamber view, three-vessel view, origin and crossing of the great arteries, as well as aortic and ductal arches. Furthermore, they recommended the transvaginal scan and the use of color-directional Doppler and power Doppler to improve the detection rate of CHD. Interestingly, Vimpelli et al. ${ }^{18}$ reported significant improvement in the visualization of the following anatomical planes: the four-chamber view, longitudinal views of the aorta and pulmonary trunks, the crossing of the great arteries, and the aortic and ductal arches from $43 \%$ at 11 th week to $62 \%$ at $13^{+6}$ week of gestation.

Over the past 10 years, the detection of fetal heart defects by obstetricians performing ultrasound screening has increased significantly. It should be taken into account that in terms of safety and highest quality of the scans, an ultrasound examination should be used according to specific recommendations and due to the up-to-date audits by an authorized and qualified physician.

\section{SUMMARY}

Taking everything into account, the fact that $\mathrm{CHD}$ remains the most important cause of infant mortality due to birth defects, and the fetal ultrasound screening enables detection of heart anomalies at an early stage of pregnancy. Currently, the ultrasonography innovations allow to diagnose CHD during pregnancy and thus improve the prognosis of most cases presenting CHDs. Many studies and guidelines on pathomechanisms of a CHD have been published. Moreover, they also present significant advances in fetal ultrasound screening in a diagnostic process of CHD and provide the tools for the detailed diagnostics of CHD by ultrasound professionals.

\section{References}

1. Horry MA. Progress in medical genetics. Nova Biomed 2006. 40-41.

2. Carvalho JS, Mavrides E, Shinebourne EA, et al. Improving the effectiveness of routine prenatal screening for major congenital heart defects. Heart 2002;88(4):387-391. DOI: 10.1136/heart.88.4.387.
3. Carvalho JS, Choui R, Copel J, et al. Cardiac screening examination of the fetus: guidelines for performing the "basic" and "extended basic" "cardiac scan". Ultrasound Obstet Gynecol 2006;27(1):107-113.

4. Bakker MK, Bergman JEH, Krikov S, et al. Prenatal diagnosis and prevalence of critical congenital heart defects: an international retrospective cohort study. BMJ Open 2019;9(7):e028139. DOI: 10.1136/bmjopen-2018-028139.

5. Liberman RF, Getz KD, Lin AE, et al. Delayed diagnosis of critical congenital heart defects: trends and associated factors. Pediatrics 2014;134(2):e373-e381. DOI: 10.1542/peds.2013-3949.

6. Hernandez-Andrade E, Patwardhan M, Cruz-Lemini M, et al. Early evaluation of the fetal heart. Fetal Diagn Ther 2017;42(3):161-173. DOI: 10.1159/000477564.

7. Pietryga M, Borowski D, Brązert J, et al. Polish Gynecological society-ultrasound section guidelines on ultrasound screening in uncomplicated pregnancy-2015. Ginekol Pol 2015;86(7):551-559. DOI: $10.17772 / g p / 58642$.

8. Sylwestrzak O, Respondek-Liberska M. Echocardiographic methods of fetal heart size assessment-heart to chest area ratio and transversal heart diameter. Prenat Cardio 2018;8(1):20-23. DOI: 10.1515/pcard2018-0003.

9. Iliescu D, Tudorache S, Comanescu A, et al. Improved detection rate of structural abnormalities in the first trimester using an extended examination Protocol. Ultrasound Obstet Gynecol 2013;42(3): 300-309. DOI: 10.1002/uog.12489.

10. Becker R, Wegner RD. Detailed screening for fetal anomalies and cardiac defects at the 11-13-week scan. Ultrasound Obstet Gynecol 2006;27(6):613-618. DOI: 10.1002/uog.2709.

11. Sinkovskaya ES, Chaoui R, Karl K, et al. Abuhamad fetal cardiac axis and congenital heart defects in early gestation. Obstet Gynecol 2015;125(2):453-460. DOI: 10.1097/AOG.0000000000000608.

12. Sinkovskaya $E$, Horton $S$, Berkley EM, et al. Defining the fetal cardiac axis between $11+0$ and $14+6$ weeks of gestation: experience with 100 consecutive pregnancies. Ultrasound Obstet Gynecol 2010;36(6):676-681. DOI: 10.1002/uog.8814.

13. Borrell A, Grande M, Bennasar M, et al. First-trimester detection of major cardiac defects with the use of ductus venosus blood flow. Ultrasound Obstet Gynecol 2013;42(1):51-57. DOI: 10.1002/uog.12349.

14. Pereira S, Ganapathy R, Syngelaki A, et al. Contribution of fetal tricuspid regurgitation in first-trimester screening for major cardiac defects. Obstet Gynecol 2011;117(6):1384-1391. DOI: 10.1097/ AOG.0b013e31821aa720.

15. Haak MC, Twisk JW, Van Vugt JM. How successful is fetal echocardiographic examination in the first trimester of pregnancy? Ultrasound Obstet Gynecol 2002;20(1):9-13. DOI: 10.1046/j.14690705.2002.00735.x.

16. Marques Carvalho SR, Mendes MC, Poli Neto OB, et al. First trimester fetal echocardiography. Gynecol Obstet Invest 2008;65(3):162-168. DOI: 10.1159/000111137.

17. Smrcek JM, Berg C, Geipel A, et al. Early fetal echocardiography: heart biometry and visualization of cardiac structures between 10 and 15 weeks' gestation. JUltrasound Med 2006;25(2):173-182.; quiz 83-85 10.7863/jum.2006.25.2.173.

18. Vimpelli T, Huhtala $H$, Acharya G. Fetal echocardiography during routine first-trimester screening: a feasibility study in an unselected population. Prenat Diagn 2006;26(5):475-482. DOI: 10.1002/pd.1442. 